Open Peer Review on Qeios

\title{
Kinesiotaping for the treatment of haematomas in haemophiliacs: a case control study
}

\author{
giulia letizia mauro ${ }^{1}$
}

1 University of Palermo

Funding: The author(s) received no specific funding for this work.

Potential competing interests: The author(s) declared that no potential competing interests exist.

\begin{abstract}
Dalila Scaturro, Fabio Vitagliani, Mariasanta Napolitano, Gianluigi Pasta, GIUSEPPINA PASSANTINO, Fausto Arcuri, Sergio Siragusa, Michele Vecchio, Giulia Letizia Mauro
\end{abstract}

Introduction: Intramuscular hemorrhages are the second most common type of bleeding in patients with haemophilia. In haemophilic patients, muscle bleeding causes pain and postural defects and requires a long healing time.

Aim: The purpose of this study is to evaluate the effectiveness of kinesiotaping, in combination with functional rehabilitation and $\mathrm{CO} 2$ laser therapy, in the healing process of $\mathrm{MH}$ in patients with haemophilia.

Methods: We recruited 39 haemophilia patients who presented with a muscle hematoma. The patients were divided into 2 groups: treatment group treated with kinesiotaping, functional re-education and $\mathrm{CO} 2$ laser therapy; and a control group treated with functional re-education and CO2 laser therapy. The evolution of the hematoma was studied ultrasonographically and some evaluation scales (NRS, FISH) were administered.

Results: The treatment group, compared to the control group, showed significant improvements in terms of: hematoma diameter $(2,36 \pm 3,32$ vs $6,47 \pm 3,37 ; p<0,05)$, hematoma thickness $(0,08 \pm 0,12$ vs $0,28 \pm 0,14 ; p=0,08$ ), pain (NRS: $2 \pm 1,57$ vs 3,53 $\pm 1,37 ; p<0,05$ ) and number of days of replacement therapy ( $n^{\circ}$ days: $1,3 \pm 0,48$ vs $3,2 \pm 0,98 ; p<0,05$ ). These results were shown to be superior in patients on prophylaxis.

Conclusions: Kinesiotaping, associated with therapeutic exercise and physical therapy, can be a valuable aid in reducing the healing times of muscle hematomas in haemophilic patients, especially if already under prophylaxis.

\section{INTRODUCTION}

Haemophilia is an inherited disease linked to the $\mathrm{X}$ chromosome resulting from a deficiency of factor VII (type A) or factor IX (type B). Based on the serum levels of the corresponding deficiency factor, we can 
distinguish several forms: mild (<1\%), moderate (1-5\%), and severe ( $>5 \%)(1)$.

After joint bleeding, muscle haematoma $(\mathrm{MH})$ is the second most common type of bleeding in patients with haemophilia $(2,3,4,5)$. In haemophilic patients, $\mathrm{MH}$ is more frequently localized in the iliopsoas muscle, in the quadriceps femoris, in the calf and forearm muscles (6).

Unlike hemarthrosis, MH is mostly associated with trauma, it is more common in young and athletic subjects (7). It is known that there is a relationship between hemophilic arthropathy and the tendency to develop $\mathrm{MH}$, as arthropathy leads to muscular and postural imbalances and incorrect movement patterns which increase the risk of injury, even after minor strains (6).

The development of a $\mathrm{MH}$, often impairs the quality of life of patients, limiting recreational activities due to the fear and/or risk of bleeding (8).

In addition, haematomas often cause pain, fibrous scars, recurrent haematomas, and postural defects, which are related to the extent of bleeding and the tension within the muscle compartment ${ }^{5}$. In haemophilic patients, muscle bleeding requires a longer healing time than haemarthroses, even if the data collected in the literature are quite scant(9).

In these patients, medical treatment of MH generally relies on intravenous infusion of the deficient factor until complete resorption. If not adequately treated, haematomas can lead to irreversible muscle damage, with reduced mobility, loss of function, ossifying myositis, anemia, compartment syndrome, and compression of peripheral nerves requiring urgent surgical decompression $(10,11)$.

To date, advances in rehabilitation, combined with hemophilia therapy, are focused on the prevention of bleeding and bleeding-relted musculoskeletal complications through targeted and specific rehabilitation programs. This allows patients with haemophilia to be more active and to participate in sports (6). The guidelines of the World Federation of Hemophilia (WFH) on the management of MH indicate that the levels of the deficiency factor should be increased immediately, after-the first symptoms or trauma, maintaining adequate factor levels for approximately 5-7 days. The affected limb must be raised and the muscle immobilized until the pain is resolved. It is necessary to establish a balance between rest, early mobilization and load restoration, to avoid the onset of complications related to prolonged immobilization and the risk of re-bleeding. To relieve pain symptoms, ice packs applied for about 15-20 minutes, several times a day, can be useful. As soon as the painful symptoms are relieved, a rehabilitation program with isometric contractions can be undertaken, followed by concentric exercises based on a symptomatic approach(12).

The effectiveness of physical therapy in the healing process of $\mathrm{MH}$ has recently been evaluated in ad hoc studies $(13,14,15)$. Among these, CO2 laser therapy has resulted effective and well tolerated in subjects with haemophilia. The $\mathrm{CO} 2$ laser is a non-invasive means, which has an anti-inflammatory and anti-edema action. It active biological mechanisms that increase blood circulation through a process that allows oxygen and nutrient-rich blood to flow to the target tissue area and improve mitochondrial oxidative metabolism(13).

The Kinesiotaping (KT) technique is an innovative technique that guarantees a rapid and decisive result in 
the resorption of the hematoma. It consists of the application of adhesive bandages in the site of the hematoma. Skin stimulation by KT determines greater recruitment of motor units with a consequent improvement in muscle strength. It also determines an increase in blood flow and an increase in interstitial spaces, favoring vascular and lymphatic drainage. Finally, by decreasing the pressure on the subcutaneous nociceptors, it modulates the intensity of muscle pain by reducing it $(14,15)$.

The purpose of this study is to evaluate the effectiveness of kinesiotaping, in combination with functional rehabilitation and $\mathrm{CO} 2$ laser therapy, in the healing process of $\mathrm{MH}$ in patients with haemophilia.

\section{MATERIAL AND METHODS}

At the Rehabilitation Department of the Polyclinic Paolo Giaccone hospital in Palermo, in collaboration with the Hematology unit of the same hospital, a single-center randomized control case study was conducted in the period between April 2020 and April 2021.

The study was approved by the Medical Ethics Committee of the University Hospital of Palermo, Italy $\left(\mathrm{n}^{\circ}\right.$ 3/2020); written informed consent was obtained from each patient by the Declaration of Helsinki.

The inclusion criteria were: age $\geq 18$ years; diagnosis of type A or B haemophilia (mild, moderate and severe); the presence of symptomatic and/or asymptomatic superficial $\mathrm{MH}$ assessed during the initial physiatric evaluation; absence of signs of nervous and/or vascular compromise; prophylactic or on-demand replacement treatment for haemophilia; written declaration of consent for participation in the study. The exclusion criteria were: altered states of consciousness and the presence of permanent deformity and/or stiffness.

A total of 54 haemophilic patients with muscle hematomas were sent, within 24 hours of observation by the haematologist, to the Rehabilitation unit to undertake a rehabilitation process. Of these, only 39 patients met the inclusion criteria and were deemed eligible to participate in the study. Through a randomized randomization process, using a computer-generated random number table, the patients were assigned to two groups:22 patients were assigned to the "treatment group" (TG) and underwent a 10session rehabilitation protocol, consisting of functional rehabilitation, $\mathrm{CO} 2$ laser therapy, and application of kinesiotaping at the hematoma site; and 17 patients were assigned to the "control group" (CG) and underwent a 10-session rehabilitation protocol consisting of functional rehabilitation and $\mathrm{CO} 2$ laser. Enrolled patients were evaluated at baseline (TO) and at the end of the 10 rehabilitation sessions, through a clinical evaluation by a physiatrist with the evaluation of demographic characteristics and administration of evaluation scales, such as NRS (16) and FISH (17). All patients finally underwent ultrasound assessment to confirm the diagnosis of $\mathrm{MH}$ and exclude another contemporary bleeding (eg in the retroperitoneal space, iliac cavity, anterior abdominal wall, and hip joint). The ultrasound examination was performed by a physiatrist with decades of experience in the musculoskeletal field. Initial images were obtained with a 7$10 \mathrm{MHz}$ linear array probe in two planes. The haematoma area was scanned from end to end to identify the deepest part and still images of this region were saved. Ultrasound evidence of a haematoma was defined as hypoechoic or anechoic changes within the subcutaneous tissue or muscle. The diameter and depth of 
the haematomas were all expressed in centimeters $(\mathrm{cm})$.

The rehabilitation program took place in 20 sessions (from Monday to Friday) lasting 60 minutes, performed on an outpatient basis in the hospital, and had the following objectives: to resolve the hematoma, to recover/maintain range of motion, to recover/maintain strength muscle, prevent joint deformity, improve posture and gait. In particular, it included joint mobilization exercises, including traction, and muscle stretching. Both methods are low-load maneuvers that allow you to maintain or restore tissue (muscles and tendons), promoting correct joint movement. Muscle strength recovery was accomplished through the initial performance of isometric exercises, followed by low-load, high-repetition exercises. Having obtained good muscle control and initial recovery, we proceeded with the execution of exercises first with an open kinetic chain and then with a closed kinetic chain, followed by concentric and finally eccentric exercises. To these was also associated a proprioceptive re-education, through oscillating tables, irregular surfaces, and visual biofeedback for the recovery of the execution of the specific gesture, as well as a global postural re-education, to improve load distribution $(18,19)$.

The lasertherapy sessions were carried out using Opton 8000scanning laser (Mectronic Medical S.r.I., Italy), under the supervision of an expert physiotherapist. The sessions were performed at the end of each functional re-education session. Every single session lasted about 10 minutes, with a wavelength used of $10,600 \mathrm{~nm}$, an emission frequency of $1-200 \mathrm{~Hz}$, and a maximum power of $20 \mathrm{~W}$. During the application of laser therapy, the physiotherapist and the patient wore protective goggles.

All the KT tape bonding procedures were performed by a physiotherapist, in possession of the K-Taping certificate. K-Active Tape Classic, $50 \mathrm{~mm} \times 17 \mathrm{~m}$ (NittoDenko Corporation, Japan) was used. These are elastic adhesive tapes $5 \mathrm{~cm}$ wide, made of $100 \%$ cotton, and covered with hypoallergenic acrylic glue applied for 3 days with the technique lymphatic drainage. The applications were carried out at the end of each rehabilitation session. Before applying the tape, the skin was cleaned, freed from moisture and grease, and, if necessary, shaved. The length of the tape was measured individually, based on the affected muscle district. The tape application procedure began with the formation of an anchor at the root of the limb, above the relevant regional lymph node stations. Further strips were attached sequentially along with the lymphatic collectors in the direction of lymphatic drainage, taking care not to create excessive tension (Figure 1).

Finally, a health worker took a photograph of the muscle haematoma at T0 and T1, in order to visually show its progress.

We used the NRS scale for pain assessment. This is a quantitative rating scale by which patients are asked to rate their pain on a defined scale, from 0 to 10(16).

The FISH scale is a valid and reliable tool for assessing the autonomy of a patient with haemophilia in carrying out activities of daily living (ADL) including self-care and transfers. Each activity is assigned a score from 1 to 4 , based on the level of independence and assistance required. The score ranges from 8 to 32, where higher scores indicate a better ability to perform ADL (17).

The primary endpoints evaluated were: the resolution of the haematoma, in terms of diameter and 
thickness; and the reduction of pain associated with haematoma.

The secondary endpoints were: the trend of the FISH scale; the number of days of treatment with coagulation Factor VIII or IX during the acute phase of the haematoma.

\section{Statistical analysis}

The data obtained were indexed on an Excel sheet and the subsequent statistical analysis was performed using the R software (R Foundation for Statistical Computing, Vienna, Austria). The descriptive analysis was performed based on the mean and standard deviation. For the statistical modeling we used the classic linear regression model to evaluate the effect net of any confounding variables. P values $<0.05$ were considered statistically significant.

\section{RESULTS}

The general characteristics of the patients, 39 male subjects, of mean age of $37.13 \pm 13.51$ years, are summarized in Table I. No statistically significant differences were noted between the two groups at baseline between the collected data.

The mean diameter of the haematomas was $15.33 \pm 6.9 \mathrm{~cm}$ and the mean thickness of $0.49 \pm 0.25 \mathrm{~cm}$. Patients reported an average pain of $5.13 \pm 1.38$, according to the NRS scale (Table II). All patients had at least one $\mathrm{MH}$ at the time of the initial clinical evaluation. 6 (15.4\%) patients had three $\mathrm{MH}$ at the time of initial clinical evaluation, 19 (48.7\%) had two, and 14 (35.9\%) patients had only one. The most common muscle localization of the haematoma was represented by the quadriceps femoris (23.1\%), forearm muscles $(20.5 \%)$, calf $(15.5 \%)$, deltoid $(12.8 \%)$, hand muscles $(10.2 \%)$, biceps brachialis $(7.7 \%)$, gluteus maximus ( $7.7 \%)$ and large pectoral (2.5\%).

Figure 2 shows the evolution of hematomas from T0 to T1, following treatment (Figure 2).

The differences in the outcomes of the variables analyzed between the treatment group and the control group at $\mathrm{T} 1$ are shown in Table III.

Regarding the diameter of the haematoma, at T1 we demonstrated a significant improvement in the treatment group compared to the control group ( $2.36 \pm 3.32$ vs $6.47 \pm 3.37 ; p<0.05)$ (Table III).

The thickness of the haematoma at $\mathrm{T} 1$ also showed a significant improvement in the treatment group compared to the control group $(0.08 \pm 0.12$ vs $0.28 \pm 0.14 ; p=0.08)$ (Table III).

A significant improvement was also demonstrated in the extent of pain associated with haematoma in the treatment group compared to the control group ( $2 \pm 1.57$ vs $3.53 \pm 1.37 ; p<0.05)$ (Table III).

Finally, no statistically significant difference in FISH scores at T1 was noted between the treatment group and the control group ( $28.3 \pm 2.31$ vs $27.7 \pm 1.88 ; p=0.40)$ (Table III).

Analyzing the number of days of treatment with recombinant Factor VIII or IX during the acute phase of the haematoma, we observed a significant reduction in the number of days of taking recombinant Factor VIII or IX in the treatment group compared to the control group ( ${ }^{\circ}$ days: $1.3 \pm 0.48$ vs $\left.3.2 \pm 0.98 ; p<0.05\right)(T a b l e$ III). 
Table IV shows, within the treatment group, the differences in the outcomes of the variables analyzed between patients in prophylactic therapy and those in on-demand therapy (Table IV).

Patients in the treatment group in prophylactic therapy were 12, while those in on-demand therapy were 10. It was observed that patients belonging to the treatment group undergoing prophylactic therapy, compared to those undergoing on-demand therapy, showed greater benefits both in terms of diameter $(1.2 \pm 0.7$ vs $5.2 \pm 3.05 ; p<0.05)$, and in thickness $(0.09 \pm 0.10$ vs $0.17 \pm 0,12 ; p=0.12)$ and both for the FISH scale score (28.9 \pm 1.14 vs $27.2 \pm 2.34 ; \mathrm{p}<0.05)$ (Table IV).

However, the improvement in pain appeared to show no significant differences in patients on prophylactic therapy compared to those on on-demand therapy ( $2.17 \pm 1.47$ vs $1.8 \pm 1.75 ; p=0.42)$ (Table IV).

\section{DISCUSSION}

Even today there is no standardized treatment protocol for the most appropriate management of MH in patients with haemophilia. This could be due to the lack of knowledge of the signs indicating that the bleeding has stopped and the scant data on treatment of MH in patients with haemophilia (5). This study aimed to evaluate the clinical effectiveness of kinesiotaping associated with therapeutic exercise and $\mathrm{CO} 2$ laser therapy in the healing process of muscle hematomas in patients suffering from haemophilia, to increase knowledge in the rehabilitation field.

$\mathrm{MH}$ is common in people with haemophilia it occurs in $10-23 \%$ of cases and can cause irreversible clinical consequences and psychosocial problems $(5,6)$. The potential long-term consequences of $\mathrm{MH}$ in haemophilia (myositis ossifying, abscess, pseudotumor, chronic nerve injury) are serious and disabling (6). It, therefore, appears clear the importance of preventing and managing these haematomas, to improve the clinical conditions of patients.

The results of our study showed how the synergy between instrumental physical therapy (CO2 laser therapy), kinesiotaping, and therapeutic exercise represents an effective therapeutic strategy in the resolution of $\mathrm{MH}$, in terms of diameter and thickness, allowing to avoid possible complications. At the end of the 10 rehabilitation sessions provided, the pain associated with haematomas also showed a clear reduction in pain, reducing the burden of the disease on patients.

Often these haematomas cause severe pain due to the increase of muscle tension, with consequent functional limitation and significant negative psychological impact. Furthermore, as a consequence of the accumulation of polymorphonuclear cells and mononuclear phagocytosis, fibrous repair tissue is formed with progressive mechanical reduction of muscle function and consequent limitation of normal joint function (5).

Over the years, attention has increased on the psychosocial problems of haemophilic patients following a bleeding episode. Participation in physical activities in haemophilic adults and children is often complicated by the risk of developing haematomas with a tendency to avoid physical activity $(7,8)$. Study B - HERO - S (20) found a negative impact on participation in recreational activities, regardless of the severity of haemophilia. The main reasons for the interruption of activities were fear/risk of bleeding or 
contact bleeding.

Our rehabilitation protocol aims to interrupt the vicious circle of haematomas-hemarthrosis and its related complications that distinguish this setting of patients. A history of arthropathy was present in about $60 \%$ of the patients examined, reflecting the high frequency of joint involvement in haemophilic patients. This supports the relationship between arthropathy and the tendency to develop $\mathrm{MH}$ (6).

In the literature, the protocol for the management of $\mathrm{MH}$ in patients with haemophilia is based on the combination of rehabilitation and replacement therapy (6).

Hematological therapy is carried out with infusion of recombinant coagulation factors deficient at $50-70 \%$ of normal during the first 5-7 days and subsequent infusion with lower doses until complete recovery (5). In our study, it was observed that the adopted protocol was able to reduce the number of days of treatment with the recombinant deficient factor during the acute phase.

The rehabilitation of $\mathrm{MH}$, through therapeutic exercise and the use of physical therapy is useful in accelerating the hematoma healing process in haemophilic patients. it aims to: control the joint and restore ROM, strength and normal physical performance $(21,22)$.

Potential benefits of physical activity for people with haemophilia include reduced joint pain and increased muscle strength and joint flexibility, as well as psychological benefits such as decreased anxiety and improved self-esteem and social interactions (8).

After the acute phase, therapeutic exercise should be introduced, to prevent joint blockages and counteract hypotonotrophy, weakness, and muscle retractions typical of these patients. An aspect that should not be underestimated is the prevention of $\mathrm{MH}$. Adequate overall physical performance helps reduce the risk of exercise-related muscle injury as deficits in muscle strength can increase the risk of muscle bleeding during physical activities. Accordingly, it is essential to perform tailored physical exercise in order to increase muscle power through resistance training (23).

De Martis et al conducted a pilot study to evaluate the safety, efficacy, and tolerability of laser therapy in hemophilic patients with chronic arthropathy. The laser treatment showed a significant analgesic effect, allowing rapid pain control to be achieved after only a few applications. The results obtained by them allow us to consider laser therapy as a possible alternative to painkiller drugs, due to its analgesic effect (24). Also in our study, laser therapy showed high safety and tolerability (no adverse reactions were registered in treated patients).

In patients with haemophilia, deficient coagulation factor prophylaxis is the standard of care for the treatment and prevention of bleeding episodes and in surgery (12). Several studies have shown that replacement therapy offered in prophylactic form offers the best results for the patient, in terms of joint health, improvement of quality of life, and reduction of bleeding episodes, compared to on-demand treatment at any age. However, adherence to continuous intravenous therapy with the deficient factor is challenging and requires good patient compliance, with the possibility of breakthrough bleeding during prophylaxis $(25,26,27,28)$.

The results of our study appear to be in line with the data in the literature. In our study, patients 
undergoing our rehabilitation on prophylaxis showed better results, in terms of size and thickness, than patients in on-demand treatment.

Moreover, in patients undergoing prophylactic treatment with the deficient recombinant factor, our rehabilitation protocol resulted in an improvement in the performance of daily life activities.

At our knowledge, this is the first case-control study to evaluate the effectiveness of the combination of functional rehabilitation, physical therapy, and kinesiotaping, compared to standard rehabilitation treatment, in the healing process of MH in patients with haemophilia. However, our study is not without limitations. Primarily, the small sample of patients, due to the rarity of the disease and the difficulty in identifying subjects who met our inclusion criteria, doesn't allow us to generalize the obtained results. A further limitation of our study was the failure to evaluate the protective effect of therapeutic exercise on the prevention of muscle hematomas. This is due to the lack of long-term follow-up investigating this effect.

\section{CONCLUSION}

Despite the advances in haematology and rehabilitation, the management of $\mathrm{MH}$ in patients with haemophilia is still matter of debate, due to the long period required for their resolution. Kinesiotaping, associated with therapeutic exercise and physical therapy, can be a valuable aid in accelerating the healing process of $\mathrm{MH}$ in haemophilic patients, especially if they are on prophylactic therapy.

This opens up new scenarios in the prognosis of these patients, strengthening the role of rehabilitation in the management of these patients.

\section{REFERENCES}

1. Bayry J, Lacroix-Desmazes S, PashovA et al. Autoantibodies to factor VIII with catalytic activity. AutoimmunRev 2003; 2: 30-5.

2. Di Minno MND, Napolitano M, Giuffrida et. Italian Association of Haemophilia Centres Musculoskeletal Working Group. Diagnosis and treatment of chronic synovitis in patients with haemophilia: consensus statements from the Italian Association of Haemophilia Centres. Br J Haematol. 2021.

3. Hilgartner MW. Current treatment of hemophilic arthropathy. CurrOpinPediatr. 2002;14(1):46-9.

4. Wessel LM, Scholz S, Rüsch M. Characteristic pattern and management of intra-articularkneelesions in differentpediatricagegroups. J PediatrOrthop. 2001;21(1):14-9.

5. Balkan C, Kavakli K, Karapinar D. Iliopsoashaemorrhage in patients with haemophilia: results from one centre. Haemophilia. 2005;11(5):463-7.

6. De la Corte-Rodriguez H, Rodriguez-Merchan EC. Treatment of musclehaematomas in haemophiliacs with special emphasis on percutaneousdrainage. Blood CoagulFibrinolysis. 2014;25(8):787-94.

7. Hernandez G, Baumann K, Knight S, et al. Ranges and drivers of riskassociated with sports and recreationalactivities in people with haemophilia: results of the Activity-Intensity-RiskConsensusSurvey of 
US physicaltherapists. Haemophilia. 2018;24 Suppl 7:5-26.

8. Baumann K, Hernandez G, Witkop M, et al. Impact of mild to severe hemophilia on engagement in recreationalactivities by US men, women, and children with hemophilia B: The BridgingHemophilia B Experiences, Results and Opportunitiesinto Solutions (B-HERO-S) study. Eur J Haematol. 2017;98 Suppl 86:25-34.

9. Mason DL, Dickens V, Vail A. Rehabilitation for hamstringinjuries. Cochrane Database Syst Rev. 2007 Jan 24;(1):CD004575.

10. Caviglia H, Candela M, Landro ME, Douglas Price AL, Neme D, Galatro GA. Haemophiliapseudotumours in patients with inhibitors. Haemophilia. 2015;21(5):681-5.

11. Fernandez-Palazzi F, Hernandez SR, De Bosch NB, De Saez AR. Hematomaswithin the iliopsoasmuscles in hemophilicpatients: the Latin American experience. ClinOrthopRelat Res. 1996;(328):19-24.

12. Srivastava A, Santagostino E, Dougall A, et al. WFH Guidelines for the Management of Hemophiliapanelists and co-authors. WFH Guidelines for the Management of Hemophilia, 3rd edition. Haemophilia. 2020;26 Suppl 6:1-158.

13. El-Shamy SM, Abdelaal AAM. Efficacy of pulsed high-intensity laser therapy on pain, functionalcapacity, and gait in children with haemophilicarthropathy. DisabilRehabil. 2018;40(4):462-468.

14. Camacho MA, Herrera E, Barela JA, Delgado-Diaz DC. Kinesiotaping Diminishes Delayed Muscle Soreness but does not Improve Muscular Performance. Int J Sports Med. 2020;41(9):596-602.

15. Aguilar-Ferrándiz ME, Castro-Sánchez AM, Matarán-Peñarrocha GA, García-MuroF, Serge T, MorenoLorenzo C. Effects of kinesiotaping on venous symptoms, bioelectrical activity of the gastrocnemius muscle, range of ankle motion, and quality of life in postmenopausal women with chronic venous insufficiency: a randomized controlled trial. ArchPhysMedRehabil. 2013;94(12):2315-2328.

16. Karcioglu O, Topacoglu H, Dikme O, Dikme O. A systematic review of the pain scales in adults: Which to use? Am J EmergMed. 2018 Apr;36(4):707-714.

17. Scaturro D, Benedetti MG, Lomonaco G, Tomasello S, Farella MGG, Frizziero A, Letizia Mauro G. Effectiveness of rehabilitation on pain and function in people affected by hemophilia. Medicine (Baltimore). 2021;100(50):e27863.

18. Klein JH. Muscular hematomas: diagnosis and management. J ManipulativePhysio/Ther. 1990 Feb;13(2):96-100.

19. Strike K, Mulder K, Michael R. Exercise for haemophilia. Cochrane Database Syst Rev. 2016 Dec 19;12(12):CD011180.

20. Blamey G, Buranahirun C, Buzzi A, et al. Hemophilia and sexual health: results from the HERO and BHERO-S studies. PatientRelatOutcomeMeas. 2019 Aug14;10:243-255.

21. Beyer R, Ingerslev J, Sørensen B. Musclebleeds in professional athletes--diagnosis, classification, treatment and potential impact in patients with haemophilia. Haemophilia. 2010 Nov;16(6):858-65. 22. Järvinen TA, Kääriäinen M, Järvinen M, Kalimo H. Muscles train injuries. CurrOpinRheumatol. 2000 Mar;12(2):155-61. 
23. González LM, Querol F, Gallach JE, Gomis M, Aznar VA. Force fluctuations during the Maximum Isometric Voluntary Contraction of the quadriceps femoris in haemophilic patients. Haemophilia. 2007 Jan;13(1):65-70.

24. Demartis F, De Cristofaro R, Fasulo M. R, Boccalandro E, Cobianco A, Santagostino E. Analgesic effects of high intensity laser therapy (hilt) for chronich emophilic artrhopathy. Energy for Health, 2013.

25. Lieuw K. Manyfactor VIII products available in the treatment of hemophilia A: an embarrassment of riches? J Blood Med. 2017; 15;8:67-73.

26. Manco-Johnson MJ, Abshire TC, Shapiro AD, et al. Prophylaxis versus episodic treatment to prevent joint disease in boys with severe hemophilia. N Engl J Med. 2007; 9;357(6):535-44.

27. Tagliaferri A, Feola G, Molinari AC, et al. POTTER Study Group. Benefits of prophylaxis versus ondemand treatment in adolescents and adults with severe haemophilia A: the POTTER study. ThrombHaemost. 2015;114(1):35-45.

28. Napolitano M, Raso S, Mansueto MF, et al. Combined Point of Care Tools Are Able to Improve Treatment Adherence and Health-Related Quality of Life in Patients with Severe Hemophilia: An Observational Prospective Study. Blood. 2019; 134 (Supplement_1): 3455. 